$\bigvee$ Workshop de Pesquisa em Manufatura

ANAIS DO V WORKSHOP DE PESQUISA EM MANUFATURA 
$\checkmark$ Workshop de Pesquisa em Manufatura

ANAIS DO V WORKSHOP

DE PESQUISA EM MANUFATURA 
Editora Omnis Scientia

ANAIS DO V WORKSHOP DE PESQUISA EM MANUFATURA

Volume 1

$1^{a}$ Edição

TRIUNFO - PE

2021 
PROMOÇÃO:

Programa de Pós-Graduação em Engenharia Mecânica (PPGEMec/UFSCar)

Apresentadores (as):

Adailton Gomes Pereira

Alana Carla Miranda Araújo

Bruna Oliveira da Silva

Bruno Alexandre Roque

Caique de Castro Gonçalves

Cassiano da Silva Tavares

Cristie Luis Kugelmeier

Gustavo Roberto dos Santos

Henrique A. C. Durello

Hugo Emanoel de Andrade Costa

Jonatan Augusto da Silva

Leones Contini Junior

Marco Gabriel Lorenzoni

Matheus Luis Manfredo

Paulo De Tarso Durigan

Rafael Fernando Teixeira

Talia Gibim

Tony Emerson Marim.

Participantes do painel:

Carlos Eiji Hirata Ventura

Danielle Cristina Camilo Magalhães

Rodrigo da Silva ("PPGEMec: Presente e futuro").

\section{Palestrante:}

Yayue Pan - University of Illinois Chicago ("Field-assisted Photopolymerization-based Additive Manufacturing for Productions of Multi-Functional Materials and Devices")

\section{Comitê científico:}

Alexandre Tácito Malavolta

Carlos Eiji Hirata Ventura

Danielle Cristina Camilo Magalhães

Flávio Yukio Watanabe

Marcos Roberto Monteiro

Rodrigo da Silva

Sérgio Henrique Evangelista.

\section{Comissão organizadora:}

Adailton Gomes Pereira 
Armando Ítalo Sette Antonialli

Bruna Oliveira da Silva

Edson Bruno Lara Rosa

Sidney Bruce Shiki.

\section{Imagem de Capa}

Freepik

\section{Edição de Arte}

Vileide Vitória Larangeira Amorim

\section{Revisão}

Os autores

\section{(C) $(1) \Theta \Theta$}

Este trabalho está licenciado com uma Licença Creative Commons - Atribuição-NãoComercialSemDerivações 4.0 Internacional.

O conteúdo abordado nos artigos, seus dados em sua forma, correção e confiabilidade são de responsabilidade exclusiva dos autores. 
Dados Internacionais de Catalogação na Publicação (CIP) (eDOC BRASIL, Belo Horizonte/MG)

Workshop de Pesquisa em Manufatura (5 : 2021)

W912a Anais do [...] / V Workshop de Pesquisa em Manufatura, 10

dezembro 2021. - Triunfo, PE: Omnis Scientia, 2021.

$52 \mathrm{p}$.

Formato: PDF

Requisitos de sistema: Adobe Acrobat Reader

Modo de acesso: World Wide Web

ISBN 978-65-88958-78-0

DOI 10.47094/978-65-88958-78-0

1. Engenharia mecânica - Brasil - Congressos. 2. Manufaturas. I. Título.

CDD 621.7

Elaborado por Maurício Amormino Júnior - CRB6/2422

\section{Editora Omnis Scientia}

Triunfo - Pernambuco - Brasil

Telefone: +55 (87) 99656-3565

$\underline{\text { editoraomnisscientia.com.br }}$

contato@editoraomnisscientia.com.br

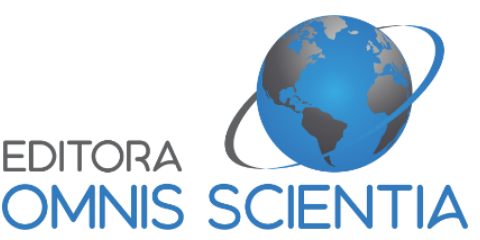




\section{PREFÁCIO}

A exemplo do ensino e da extensão, a pesquisa no âmbito da Engenharia Mecânica apresenta-se bastante ampla e diversificada. A ramificação mais canônica dessa modalidade de engenharia aponta para três áreas fundamentais: Projeto de Sistemas Mecânicos, Ciências Fluidotérmicas e Processos de Fabricação. Outras subdivisões, como Dinâmica e Vibrações, Mecatrônica e Metrologia, flutuam entre as três grandes áreas. Sendo assim, a nucleação e o crescimento do Grupo de Pesquisa em Manufatura Inteligente (GPMI), devidamente registrado no Diretório dos Grupos de Pesquisa do CNPq e reconhecido pela UFSCar, têm sido salutar no sentido da consolidação do Departamento de Engenharia Mecânica (DEMec) e do curso de graduação em Engenharia Mecânica. Da mesma forma, O GPMI se mostra essencial enquanto alicerce para o recém-criado curso de mestrado acadêmico dentro do Programa de PósGraduação em Engenharia Mecânica (PPGEMec), conferindo-lhe vocação e identidade. Finalmente, a comunicação e a difusão dos resultados obtidos pelo grupo propiciam o alinhamento do mesmo ao lema da UFSCar: excelência acadêmica e compromisso social. O objetivo geral do "V Workshop de Pesquisa em Manufatura" consiste em disseminar os trabalhos em andamento dentro do Grupo de Pesquisa em Manufatura Inteligente (GPMI) e de outros grupos afins à área de Manufatura, promovendo discussões

profícuas e de alto nível com o público interno e externo à universidade. Como objetivos específicos, destacam-se a exposição dos estudantes de graduação e pós-graduação a um ambiente de conferência científica sem sair de casa e a oportunidade de contar com convidados com grande potencial de contribuição a essa área do conhecimento. O evento foi realizado ao longo do dia 10 de dezembro de 2021, de forma online, de maneira a viabilizar e fomentar a participação de uma parcela significativa de estudantes. 


\section{SUMÁRIO}

INFLUÊNCIA DA SENSIBILIDADE DE PARÂMETROS DOS MODELOS DE AVRAMI NAS CINÉTICAS DE RECRISTALIZAÇÃO SOBRE O TAMANHO DE GRÃO EM UM PROCESSO DE LAMINAÇÃO A QUENTE DE LIGA DE AÇO SAE 4140..

SIMULAÇÃO NUMÉRICA DA EXTRUSÃO EM CANAL ANGULAR COM TORÇÃO (ECA-T): INFLUÊNCIA DA GEOMETRIA NA DEFORMAÇÃO.

OTIMIZAÇÃO TOPOLÓGICA APLICADA NO CONTEXTO DE PRÓTESES HUMANAS. .15

PROCESSO DE DOBRAMENTO A FRIO DE CHAPAS FINAS METÁLICAS: ANÁLISES POR ELEMENTOS FINITOS, EXPERIMENTOS E MODELO MATEMÁTICO PARA COMPENSAÇÃO DO RETORNO ELÁSTICO.

FORÇAS NA ESTAMPAGEM INCREMENTAL DE CHAPA FINA METÁLICA POR MEIO DE EXPERIMENTOS E ANÁLISES POR ELEMENTOS FINITOS.

MODELAGEM DE CURVAS DE FLUXO PLÁSTICO DE UM AÇO BIFÁSICO UTILIZANDO INTELIGÊNCIA ARTIFICIAL

APLICAÇÃO DA TECNOLOGIA DE MACHINE LEARNING PARA AVALIAÇÃO DA QUALIDADE DE PEÇAS IMPRESSAS EM 3D...

ESTUDO DA PREVISIBILIDADE DO ERRO DE TRANSMISSÃO DE ENGRENAGENS DE DENTES RETOS PARA PROJETO DE MODIFICAÇÕES DE MICROGEOMETRIA...........................26

CONTRIBUIÇÃO DO PRÉ-AQUECIMENTO DO SUBSTRATO NA PREVENÇÃO DE TRINCAS EM REVESTIMENTOS GERADOS POR LASER CLADDING.

AVALIAÇÃO DA INFLUÊNCIA DA VELOCIDADE DE CORTE SOBRE O ESTADO DE SUPERFÍCIE NO TORNEAMENTO DA LIGA TI-6AL-4V ELI.

INFLUÊNCIA DO TAMANHO DO GRÃO DO REBOLO NA QUALIDADE SUPERFICIAL DO INSERTO DE CORTE DE METAL DURO RETIFICADO.

INVESTIGAÇÃO DOS MECANISMOS DE REMOÇÃO DE MATERIAL NA RETIFICAÇÃO DE INSERTOS DE CORTE.

SHUNT PIEZOELÉTRICO PARA CONTROLE PASSIVO DE CHATTER NO PROCESSO DE TORNEAMENTO DE LIGAS DE TITÂNIO.

AVALIAÇÃO DE TEXTURAS INDUZIDAS POR VIBRAÇÃO NO TORNEAMENTO DURO DE UM AÇO FERRAMENTA.

INVESTIGAÇÃO DA INFLUÊNCIA DO CONTROLE TÉRMICO ATIVO SOBRE A RESISTÊNCIA MECÂNICA E QUALIDADE SUPERFICIAL DE PEÇAS IMPRESSAS PELA TÉCNICA FPM. 
ATUALIZAÇÃO DE MÁQUINAS-FERRAMENTA ANTIGAS ATRAVÉS DE INSTRUMENTAÇÃO DE BAIXO CUSTO: UM ESTUDO SOBRE O EFEITO DO MONITORAMENTO DO PROCESSO DE TORNEAMENTO NA QUALIDADE SUPERFICIAL .46 AVALIAÇÃO MICROESTRUTURAL DO AÇO INOXIDÁVEL LEAN DUPLEX 2404 APÓS SIMULAÇÕES DE CICLOS TÉRMICOS DE ZONAS TERMICAMENTE AFETADAS REALIZADAS POR GLEEBLE. . .48 AVALIAÇÃO DA INFLUÊNCIA DO ACABAMENTO SUPERFICIAL NA RESISTÊNCIA À CORROSÃO DO AÇO 1020 EM BIODIESEL E SUAS MISTURAS COM ÓLEO

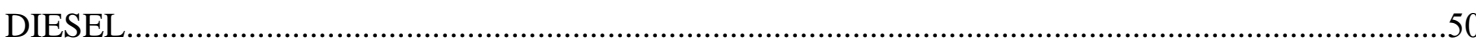




\title{
PPGEMec Programa de Pós-Graduação em

\section{PREVISÃO DE FORÇAS NA ESTAMPAGEM INCREMENTAL DE CHAPA FINA METÁLICA POR MEIO DE EXPERIMENTOS E ANÁLISES POR ELEMENTOS FINITOS}

\author{
Tony Emerson Marim ${ }^{(1)}$ \\ Sérgio Henrique Evangelista ${ }^{(2)}$ \\ (1) Mestrando, Universidade Federal de São Carlos, Brasil, marim.tonyemerson@gmail.com \\ (2) Professor Dr. Universisdade Federal de São Carlos, Brasil, toddyprof@ufscar.br
}

Resumo: No presente trabalho é investigado o comportamento das chapas de alumínio de uso industrial QQ-A-250/5 'T3' (2024), com 0,40mm de espessura, quando exposto ao processo de Estampagem Incremental (EI). Especificamente para a pesquisa, foi utilizada a modalidade de EI de Ponta Única com uma ferramenta de ponta semiesférica. Foi elaborado um modelo em Elementos Finitos (EF) por meio do uso do software ABAQUS (Dassault Systèmes) com dados materiais colhidos anteriormente em ensaio de tração, possibilitando aproximar na simulação os valores adquiridos nos experimentos reais. Experimentos e análises por EF foram executados sobre alguns casos que combinam raio da ponta de ferramenta e passo diferentes. Foi feito o cálculo das forças de processo por EF e estes valores foram comparados com um modelo matemático ajustado por outros autores sobre os parâmetros do processo para a liga de alumínio utilizada. Mostrou-se que com baixo tempo de processamento computacional com os presentes modelos foi possível obter uma previsão de forças cujos erros em relação ao modelo da literatura ficaram enquadrados em uma faixa restrita de previsão. Isto aponta para a viabilidade da técnica de previsão discutida às aplicações industriais com peças mais complexas.

Palavras-chaves: Estampagem incremental, Alumínio, Elementos finitos, Conformação de chapas.

\section{INTRODUÇÃO}

$\mathrm{Na}$ Estampagem Incremental (EI), assim como nos processos mecânicos convencionais, devem ser considerados os diversos parâmetros a serem analisados durante sua aplicação.

Este artigo apresenta uma avaliação da influência das ações das forças na EI do tipo de ponta única, com ferramenta de ponta semiesférica rotativa simulada em um modelo elaborado em Elementos Finitos (EF), a fim de prever as ocorrências na deformação obtida na estampagem de chapas finas do alumínio 2024.

\section{MATERIAIS E MÉTODOS}

A estampagem foi feita em chapas de Alumínio QQA-250/5 'T3' (2024) com dimensões de 0,4 x 77 × 127 $\mathrm{mm}$. Para cada experimento foi determinado o percurso e o incremento das ferramentas por programação em uma máquina CNC da marca Romi e modelo D800.

Em todos os experimentos foi desenvolvida uma geometria cônica e em espiral, sendo mantidos a rotação da ferramenta em 1000 RPM e um avanço constante de F $=200 \mathrm{~mm} / \mathrm{min}$. sobre a chapa nos eixos X, Y e Z, por meio de ferramenta semiesférica. $O$ incremento vertical inicial sobre o eixo $\mathrm{Z}$ iniciou-se em $0,5 \mathrm{~mm}$, percorrendo uma forma circular com profundidade de $15 \mathrm{~mm}$, mantendo um ângulo de $45^{\circ}$ de parede em relação a ferramenta.

O modelo para análises em EF foi construído com o auxílio do software ABAQUS, para analisar a influência do atrito, das tensões e forças aplicadas durante a EI, fixando-se a malha e variando-se as condições de contorno.

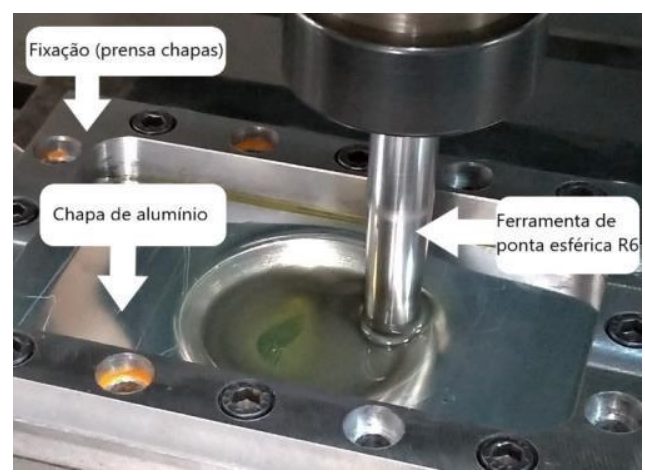

Figura 1: Experimento de EI.

As ferramentas foram simplificadas em formas semiesféricas e assim como o prensa-chapas foram determinadas como elementos rígidos.

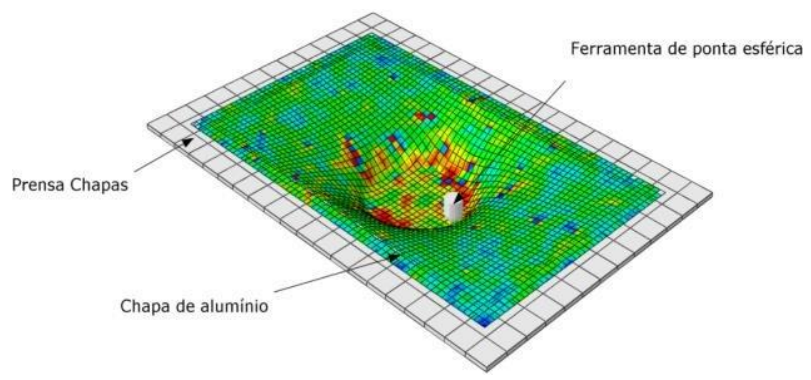

Figura 2: Modelo do EF. 
Para análise da malha foram levadas em consideração as forças obtidas no final da simulação e as análises feitas nos elementos escolhidos conforme a realização do trajeto da ferramenta com a superfície da chapa. Na predição dos valores da força no eixo Z Fz, Aerens et al. (2010) desenvolveram a variável dependente $F_{s}$, que é a força em $\mathrm{Z}$ dada em Newton.

$$
F_{s}=0,0716 R m t^{1,57} d t^{0,4} \Delta h^{0,09} \alpha \cos \alpha
$$

em que, $R m$ é o valor de resistência a tração $\left(\mathrm{N} / \mathrm{mm}^{2}\right), t$ é a espessura da chapa $(\mathrm{mm}), d t$ é o diâmetro da ferramenta (mm), $\alpha$ é o valor do ângulo da parede e $\Delta h$ é altura do scallop relacionado ao incremento de profundidade $\Delta h^{1}$.

$$
\Delta h=2 \sin \alpha \sqrt{\Delta h^{1}\left(d t-\Delta h^{1}\right)}
$$

\section{RESULTADOS PRELIMINARES}

No aspecto final das chapas extraídas em 2 casos que combinam raio da ferramenta e passos diferentes, nota-se o detalhe da Fig. 3 com a indicação da abertura da trinca, o que condicionou a interrupção do processo.

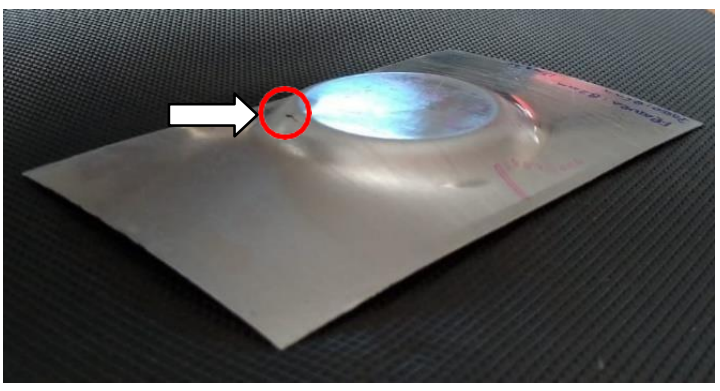

Figura 3: Trincas no experimento.

A Fig. 4 ilustra em superposição as curvas de forças nas direções $\mathrm{X}, \mathrm{Y}$ e $\mathrm{Z}$, bem como os deslocamentos na direção Z, para o caso de EI em que a ferramenta tinha raio de $3 \mathrm{~mm}$, com passos de 0,20 mm. Esta configuração refere-se ao caso experimental demonstrado na figura 3 . Não serão demonstradas as curvas para os outros casos. Elas foram obtidas numericamente para um deslocamento de $15 \mathrm{~mm}$, mas os dados efetivos de forças contemplaram uma região de análise dos gráficos em que os deslocamentos eram menores ou iguais aos efetivos em seus respectivos experimentos.

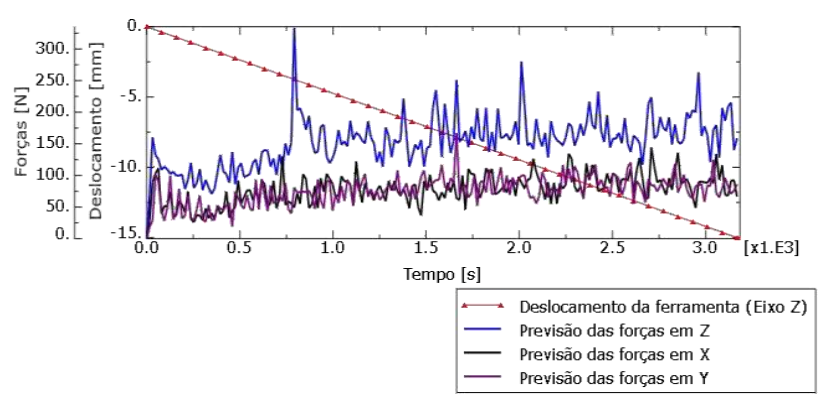

Figura 4: Previsão das forças na EI.

A previsão de esforços nos vários casos demonstrou uma grande oscilação das cargas. É possível que isto seja uma penalização de caráter numérico devido ao fato de que simplificações foram adotadas na implementação do modelo de EF para que as análises durassem em torno de 20 minutos para serem executadas.

A Tab. 1 traz um comparativo das forças aplicadas ao eixo Fz a partir da estimativa pela Eq.(1) dos autores e por EF. Observa-se que foi desconsiderada neste estudo a influência da rotação da ferramenta pois a presença dos valores de rotação empregados nas simulações em comparação com os cálculos realizados implicaram em uma redução média de apenas $5 \%$ da força final no eixo $\mathrm{Z}$ (AL-OBAIDI; KRÄUSEL, 2016).

Observa-se que os valores obtidos por EF ficaram em geral menores que os valores obtidos da Eq.(1) Verificase que o módulo de erros de previsão ficou abaixo de $30 \%$.

Dos valores obtidos por EF destacou-se na Tab. 1 o pico mais pronunciado em cada caso.

Tabela 1: Comparação dos valores do experimento.

\begin{tabular}{ccccc}
\hline $\begin{array}{c}\text { Raio da } \\
\text { ferramenta }\end{array}$ & $\begin{array}{c}\text { Passo } \\
\text { (incremento) }\end{array}$ & $\begin{array}{c}\text { Valores } \\
\text { estimados } \\
\text { conforme Eq. } \\
\text { (1) }\end{array}$ & $\begin{array}{c}\text { Valores obtidos por } \\
\text { Elementos finitos }\end{array}$ & $\begin{array}{c}\text { Erro \% em relação } \\
\text { ao estimado pela } \\
\text { Eq. (1) }\end{array}$ \\
\hline R $6 \mathrm{~mm}$ & 0,5 & $392,2 \mathrm{~N}$ & $328 \mathrm{~N}$ & $-16,4 \%$ \\
R $3 \mathrm{~mm}$ & 0,5 & $314,2 \mathrm{~N}$ & $311 \mathrm{~N}$ & $-1,01 \%$ \\
R $6 \mathrm{~mm}$ & 0,2 & $332,6 \mathrm{~N}$ & $233 \mathrm{~N}$ & $-29,9 \%$ \\
R $3 \mathrm{~mm}$ & 0,2 & $266,4 \mathrm{~N}$ & $234 \mathrm{~N}$ & $-12,2 \%$ \\
\hline
\end{tabular}

Por outro lado, das estimativas obtidas pela Eq. (1), consideram-se as investigações feitas pelos autores da equação. Estes ponderaram pelo fato de que as equações apresentadas em seu trabalho para vários materiais referiram-se às curvas ajustadas a partir de uma quantidade grande de experimentos, com variações em suas condições. Disto, para a liga de Alumínio 2024 foi estimado que o erro de previsão da equação em relação aos seus experimentos ficou entre $+10 \%$ e $-24 \%$. Estes autores trabalharam com ferramentas de raio maior ou igual a $10 \mathrm{~mm}$.

Os erros de predição de força neste trabalho poderiam ser estimados a partir de uma composição entre os valores da Tabela 1 e os valores obtidos pela Eq. (1) para a liga de alumínio utilizada. Assim, estima-se que o padrão de implementação por EF empregado neste trabalho deverão produzir erros compostos em relação a experimentos futuros que fique na faixa entre $+9 \%$ e $-45 \%$.

Considerações adicionais podem ser feita acerca de melhorias ao modelo de EF empregado e que possam contribuir com uma previsão mais robusta dos esforços desejados.

\section{CONCLUSÃO}

Erros de predição da força em $\mathrm{Z}$ para o estudo foram estimados para ficarem entre $+9 \%$ e $-45 \%$ quando comparado com trabalho de outros autores. A condição de implementação dos presentes modelos mediante esta faixa de erros de predição sugere a busca por melhorias discutidas. Dentre elas, destacam-se a melhoria, no âmbito do modelo de EF, sobre as condições de travamento da borda da chapa na matriz e sobre a escolha do modelo de atrito e seu coeficiente. Vale enfatizar a importância em se 
manter o tempo de processamento computacional baixo (20 minutos) para que a disseminação em âmbitoindustrial da técnica seja atrativa, mesmo com erros de predição enquadrados na faixa acima citada.

\section{REFERÊNCIAS}

Aerens, R., Eykens, P., Bael, A.P., e Duflou, J.R., 2010.

"Force prediction for single point incremental forming deduced from experimental and fem observations.". The International Journal of Advanced Manufacturing Technology, Vol.46, No. 9, pp. 969-982.

Al-Obaidi, A., Kräusel, V., e Landgrebe, D., 2016. "Hot single-point incremental forming assisted by induction heating". International Journal of Advanced Manufacturing Technology, No. 82, pp. 1163-71. 
editoraomnisscientia@gmail.com M https://editoraomnisscientia.com.br/ @editora_omnis_scientia https://www.facebook.com/omnis.scientia.9

+55 (87) 9656-3565 


\section{EDITORA

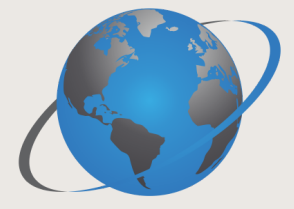 \\ OMNIS SCIENTIA}

editoraomnisscientia@gmail.com M https://editoraomnisscientia.com.br/ $\circledast$

@editora_omnis_scientia (0)

https://www.facebook.com/omnis.scientia.9 f

$$
\text { +55 (87) 9656-3565 }
$$

